\title{
A CORRESPONDENCE PRINCIPLE FOR MOMENTUM OPERATORS
}

\author{
J. E. Marsden \\ (received October 1, 1966)
}

1. Introduction. The purpose of this note is to give a precise formulation of the correspondence principle between classical and quantum mechanics, for the case of momentum operators. (See e.g. I. Segal, Journ. Math. Phys. (1960), 475).

We follow Abraham - Marsden [1] for notation.

2. Induced Vectorfields. Let $M$ be a manifold (configuration space) and $T^{*} M$ the cotangent bundle (phase space) with the natural symplectic structure, $[1, \S 14]$. Classical mechanics deals with Hamiltonian vectorfields on $T^{*} \mathrm{M}$, while quantum mechanics is concerned with linear operators on $\mathcal{F}(\mathrm{M})$, the smooth functions on $M$. Notice that if $X$ is a vectorfield on $M$, then $i L_{X}$ may be regarded as a self adjoint operator; (see $[1]$, exercises for $\$ 12$ ).

PROPOSITION. If $F_{t}$ is a flow on $M$, then $F_{t}^{*}$ is a flow on $\mathrm{T}^{*} \mathrm{M}$.

Proof. Clearly $\mathrm{F}_{\mathrm{t}}^{*}$ is a diffeomorphism and

$$
F_{t+t^{\prime}}^{*}=\left(F_{t^{\prime}} \circ F_{t^{\prime}}\right)^{*}=F_{t}^{*} \circ F_{t^{\prime}}^{*}
$$

which proves the assertion $[1, \S 10]$.

DEFINITION. If $X$ is a vectorfield on $M$, with (local) flow $F_{t}$ let $X^{*}$ be the vectorfield on $T^{*} M$ generating $F_{t}^{*}$.

PROPOSITION. $\quad X$ is locally Hamiltonian $[1, \S 16]$.

Canad. Math. Bull. vol. 10, no. 2, 1967 
Proof. From [1, $\$ 14], F_{t}^{*}$ is a symplectic diffeomorphism, and hence the corresponding vectorfield is locally Hamiltonian.

Thus we may write, locally, $X^{*}=X_{P}$ for some smooth function $P$.

3. Momentum Operators.

DEFINITION. Suppose $X$ is a vectorfield on $M$ with corresponding vectorfield $X^{*}$ on $T^{*} M$. Suppose that $X^{*}$ is globally Hamiltonian. (It is easy to show that this always holds). If $X^{*}=X_{P}$ we say that $P$ is the classical momentum corresponding to the quantum operator $i L_{X}$. (Lie derivative.)

PROPOSITION. Let $f$ be a smooth function on $M$ and $f^{*}=p^{*} f=f \circ p$, where $p: T^{*} M \rightarrow M$ denotes the canonical projection. Then

$$
p^{*} L_{X} f=L_{X} * p^{*} f
$$

This follows easily from the fact that

$$
p^{*}\left(f \circ F_{t}\right)=\left(p^{*} f\right) \circ F_{t}^{*}
$$

PROPOSITION. Restricted to functions on $M$, we have

$$
\left[x_{1}^{*}, x_{2}^{*}\right]=\left[x_{1}, x_{2}\right]^{*}
$$

for any two vectorfields $x_{1}$ and $x_{2}$ on $M$.

This follows immediately from the above proposition.

4. Correspondence Principle. Our main result may be stated as follows:

THEOREM. Let $P_{1}$ and $P_{2}$ be classical momenta corresponding to quantum operators $i X_{1}$ and $i X_{2}$ respectively. Then, restricting to functions on $M,\left\{P_{1}, P_{2}\right\}$ (the classical Poisson bracket) is the classical momentum corresponding to the 
quantum operator $i\left[x_{1}, x_{2}\right]$.

Proof. From $[1, \S 14]$ we have $L_{\left[x_{P^{\prime}}, x_{Q}\right]}=-L_{x} x_{\{P, Q\}}$ ' from which the result follows by the above proposition.

5. Position Operators. As we have seen, for each $f \in \mathcal{F}(\mathrm{M})$ there is associated $f^{*} \in \mathcal{F}\left(T^{*} M\right)$ in a natural way. Interpreting $f$ as an operator on $\mathcal{F}(\mathrm{M})$, namely multiplication by $f$, we say that $f^{*}$ is the corresponding classical position. The correspondence principle extends to this case as follows:

THEOREM. If $f, g \in \mathcal{F}(M)$ then $\left\{f^{*}, g^{*}\right\}=0$ is the classical position corresponding to the operator $[f, g]=0$. (trivial.) If $P$ is the classical momentum corresponding to a vectorfield $X$ on $M$, and $f \varepsilon \mathcal{F}(M)$ then $\left\{P, f^{*}\right\}$ is the classical position corresponding to the operator $-L_{X} f=-X(f)$.

Proof. From $[1, \S 14]$, we have $\left\{P, f^{*}\right\}=-L_{X_{P}} f^{*}=$ $-X^{* *}\left(f^{*}\right)=-X(f)^{*}$; the latter equality following from 3 above. This proves the result.

Much of the above carries through for distributions (in the sense of Schwartz) as well as that in [1]. These results will be given in the near future [3].

6. Remarks. It is shown in [1] that the usual linear and angular momenta correspond to the linear and angular momentum operators in quantum mechanics, in the sense we have defined here. Hence it is no surprise that both satisfy the same commutation rules. It is easy to see that $P\left(\alpha_{m}\right)=\alpha_{m} \cdot X(m)$. See Sternberg [4], p.146. This is exploited in [3].

\section{REF ERENCES}

1. Abraham - Marsden, Foundations of Classical Mechanics, Benjamin, New York (1967).

2. Mackey, Mathematical Foundations of Quantum Mechanics. Benjamin, New York (1963). 
3. Marsden, Mathematical Foundations on Continuum and Distributional Mechanics (to appear).

4. Sternberg, Lectures on Differential Geometry. PrenticeHall, N.J. (1964).

Princeton University 